\title{
Clinical Study of The Early Application of Lvabradine For Acute Anterior Myocardial Infarction Patients After PCI With Early Heart Failure To Observe Cardiac Function And Prognosis
}

\section{Jun-Qing Gao}

Shanghai University of Traditional Chinese Medicine

Xu wang ( $\nabla$ dwx_1234@126.com )

Shanghai University of Traditional Chinese Medicine https://orcid.org/0000-0002-5854-6060

\section{Ling-Yan Li}

Shanghai University of Traditional Chinese Medicine

\section{Hua Zhang}

Shanghai University of Traditional Chinese Medicine

\section{Hong Zhang}

Shanghai University of Traditional Chinese Medicine

\section{Ying Liu}

Shanghai University of Traditional Chinese Medicine

Tian-Jiao Lv

Shanghai University of Traditional Chinese Medicine

\section{Bing-Yu Han}

Shanghai University of Traditional Chinese Medicine

\section{Ye Jian}

Shanghai University of Traditional Chinese Medicine

\section{Zong-Jun Liu}

Shanghai University of Traditional Chinese Medicine

\section{Study protocol}

Keywords: Ivabradine, acute myocardial infarction, cardiac function

Posted Date: June 28th, 2021

DOl: https://doi.org/10.21203/rs.3.rs-516241/v1

License: (a) This work is licensed under a Creative Commons Attribution 4.0 International License. Read Full License 
Page $2 / 13$ 


\section{Abstract}

\section{Background}

The incidence of acute myocardial infarction is increasing each year. Percutaneous coronary intervention has become highly preferred for patients with acute myocardial infarction because it not traumatic and improves heart function. However, the mortality and disability rates are still high. For the first time, we used ivabradine in patients with acute anterior myocardial infarction. We expect that this new method will enhance heart function and clinical prognosis because of heart rate control, decreases in heart preload and improvements in left ventricular end-diastolic volume.

\section{Method and analysis}

This is a prospective, randomized, controlled, open-label, multicenter and optimally designed clinical trial. A total of 500 patients with acute anterior myocardial infarction after Percutaneous coronary intervention $\triangle \mathrm{PCI} \varangle$ with early heart failure will be enrolled. Eligible subjects will be randomized at a 1:1 ratio to take the standard drug treatment or receive the standard drug treatment plus ivabradine. The primary outcome measure is left ventricular end-diastolic volume. Left ventricular ejection fraction, adverse cardiac events, and the Canadian angina pectoris score will be evaluated as secondary endpoints. Blood biochemical testing will be used as the safety endpoints.

\section{Ethics and dissemination}

The clinical research will be carried out in strict accordance with the relevant Chinese laws and regulations, the Declaration of Helsinki, and the ethical and scientific principles stipulated by the Chinese GCP. All participants will provide informed consent. The personal information of patients will be kept confidential. Findings from the trial will be disseminated through peer-reviewed journals and scientific conferences.

ClinicalTrials.govID:ChiCTR2000032731,Registered8May,2020 http://www.chictr.org.cn/showproj.aspx? proj $=53275$

\section{Trial Status}

version number: Protocol version 1.0., approved9 May $₫ 2020$

\section{Trial ongoing}

Study execute time: From 1 September 2020 to 31 Octomber 2022

Recruiting time: From 8 May 2020 to 31 December 2022

\section{Background}


Acute myocardial infarction (AMI) is increasing each year and the population affected tends to be younger. AMI has become a serious global public health problem, contributing to patient mortality and disability. The development of percutaneous coronary intervention (PCl) technology has significantly reduced the mortality rate, but the fatality and disability rates are still relatively high. Thus, it is worthwhile for researchers to explore a treatment to reduce fatality and disability rates.

Currently, $\beta$ receptor blockers are used as recommended IA antiarrhythmic drugs to control the heart rate of acute myocardial infarction patients. However, because these drugs reduce blood pressure and exert negative effects on cardiac contractility, atrioventricular conduction function, and bronchial smooth muscle, their clinical application is limited, especially in decompensated heart failure patients. Moreover, a meta-analysis ${ }^{1}$ showed that the clinical benefits of $\beta$ receptor blockers in acute myocardial infarction patients is related to heart rate, but not to the doses of $\beta$ receptor blockers. Therefore, it may be possible to find a new drug to replace $\beta$ receptor blockers. We expect that a new treatment method would improve the clinical prognosis of acute myocardial infarction patients by reducing heart rate.

Ivabradine has emerged as a novel antiarrhythmic drug in recent years that selectively inhibits the fourphase automatic depolarization rate of the sinus node in a dose-dependent manner and slows the sinus rhythm. Because it binds to the If channel of sinoatrial node cells and specifically inhibits the If current, ivabradine reduces depolarization speed and heart rate. Ivabradine also has no direct effect on myocardial contraction, ventricular repolarization or intracardiac conduction time ${ }^{2}$.

Ivabradine is currently recommended for use in heart failure patients and angina pectoris patients. The SHIFT study ${ }^{3}$ laid a strong foundation for the application of ivabradine in heart failure with low ejection fraction. In this study, if patients received a conventional anti-heart failure drug treatment plus ivabradine, there was an $18 \%$ reduction in the primary composite endpoint, a $26 \%$ reduction in mortality, and a $26 \%$ reduction in hospitalization because of heart failure. The INTENSIFY study ${ }^{4}$ observed $1956 \mathrm{HFrEF}$ patients. After 4 months of follow-up, the heart rates of patients in the ivabradine group were slowed and the levels of brain natriuretic peptide were significantly reduced. In addition, the NYHA heart function classification was significantly increased. Furthermore, the "2018 Chinese Guidelines for the Diagnosis and Treatment of Heart Failure" 5 also clearly pointed out that if the heart rate of heart failure patients was $\geq 70 \mathrm{bpm}$ after the use of $\beta$-blockers, it was recommended to use ivabradine to control the ventricular rate.

The application of ivabradine in acute myocardial infarction patients is still in the exploratory stage. Barillà ${ }^{6}$ studied acute ST-segment elevation myocardial infarction and cardiogenic shock patients in whom early application of ivabradine led to a downward trend in mortality and improved left ventricular ejection fraction, left ventricular diastolic function, NT-pro BNP and other indicators during hospitalization. In a clinical study of 468 inferior myocardial infarction patients, Priti $\mathrm{K}^{7}$ showed that there was no difference in the incidence of third degree atrioventricular block, myocardial remodeling or cardiac function between the metoprolol group and the ivabradine group. However, the incidences of first and second degree atrioventricular block were significantly higher in the metoprolol group than in the 
ivabradine group. In the ischemia-reperfusion mouse model, O'Connor DM ${ }^{8}$ showed that ivabradine could significantly improve ventricular remodeling by reducing the ventricular rate and heart rate variability. In a myocardial infarction and diabetes rat model, Cao $X{ }^{9}$ showed that ivabradine could significantly reduce the uptake of norepinephrine in the myocardium and the plasma level of brain natriuretic peptide. In addition, ivabradine has a significant effect on ventricular function. Cardiac remodeling plays an important role in improving the systolic function of the heart. Previous research also showed that ivabradine could significantly reduce the heart rate of myocardial infarction patients and the levels of NTpro BNP and troponins.

In summary, even if the blood supply channel of acute myocardial infarction patients has been successfully reopened, the increased ventricular rate still affects the clinical prognosis of the patients. When Ivabradine reduces the heart rate but does not lower blood pressure or affect the contractility of the heart. The research team believes that ivabradine may be a promising treatment method that can reduce the mortality rate of early acute myocardial infarction and improve the clinical prognosis of patients with myocardial infarction.

\section{Methods/design}

\section{Study design}

This is a controlled, prospective, national, multicenter and randomized research project. Approximately 10 clinical centers, including 500 acute anterior myocardial infarction cases, will participate in this trial. There will be 250 cases in each group with a total follow-up of 6 months.

\section{Inclusion criteria}

(1) Subjects with acute anterior myocardial infarction who have received emergency $\mathrm{PCl}$ treatment.

(2) ECG shows sinus rhythm, and ventricular rate $\geq 75$ beats $/ \mathrm{min}$.

(3) Subject is $\geq 18$ and $<85$ years old at the time of randomization.

\section{Exclusion criteria}

(1) Subject has a history of bronchial asthma.

(2) Subject has a history of bradyarrhythmia.

(3) Systolic blood pressure $\leq 90 \mathrm{mmHg}$.

(4) Subject uses vasoactive drugs because of shock 6 hours after PCI.

\section{Elimination criteria}

(1) Subjects did not follow the study protocol. 
(2) Investigators consider it necessary to terminate the study of subjects from the perspective of medical ethics, including that the condition of subjects has worsened after medication.

(3) Subjects withdraw from the trial, are lost to follow-up or die because of various other reasons.

(4) The case data are incomplete and affect the judgment of effectiveness and safety.

\section{Randomization process}

After PCl, patients who meet the criteria will be divided into two groups according to the randomization method. Eligible subjects will be randomized at a 1:1 ratio to undergo standard drug treatment or to receive the standard drug treatment plus Ivabradine. A stratified block randomization with randomly varying block sizes will be performed and will be stratified according to study site. Random allocation will be generated by independent statisticians and implemented by random envelopes assigned to each site. These envelopes will be opaque and have no information to identify processing assignments. No one shall open the envelope unless there is a real eligible subject requiring randomization. To avoid potential selection bias, the sequence will be hidden from both investigators and patients until distribution. Therefore, neither the investigators nor the participants can influence which group the study patients are assigned to.

\section{Sample size estimation}

$n_{1}=n_{2}=2 \times\left[\frac{\left(u_{\alpha}+u_{\beta}\right) \sigma}{\delta}\right]^{2}$

It has been previously reported that the main outcome (left ventricular end diastolic volume) differs approximately $6 \mathrm{ml} / \mathrm{m} 2$ between the experimental and the control groups. The standard deviation differs approximately $5.0 \mathrm{ml} / \mathrm{m} 2$. With a sample size of 112 randomized patients per group, the between-group comparison will be powered at $80 \%$ to establish the superiority of added ivabradine for the primary endpoint at a two-sided significance level of 0.05 . Given an expected dropout rate of $10 \%$, a total of 500 patients (246 patients per group) must be enrolled in the study.

\section{Intervention}

After percutaneous coronary intervention, all patients will be divided into two groups according to the random number method. One group will undergo conventional treatment, and the other will undergo conventional treatment plus ivabradine. If patients are assigned to the ivabradine group, ivabradine should be orally administered twice a day at a dose of $5 \mathrm{mg}$ each time within 24 hours. Every patient should undergo standard drug treatment, including aspirin, ticagrelor or clopidogrel (antithrombotic therapy), statins (treatment to stabilize cardiac plaques and lower lipids), ACEl or ARB inhibition (myocardial remodeling treatment) and $\beta$-receptor blockers without contraindications. Investigators will 
change the dose of $\beta$-blockers according to the "2015 Guidelines for the Diagnosis and Treatment of Acute Myocardial Infarction (Table 1).

\section{Primary endpoints}

(1) Cardiac ultrasound examination: the first day, the seventh day and the sixth month after operation.

Index includes ejection fraction, left ventricular end-diastolic diameter, left ventricular end-systolic diameter, left atrial inner diameter, left atrium volume index, left ventricular posterior wall thickness, ventricular septal thickness, left ventricular end-systolic volume, left ventricular end-diastolic volume and mitral valve annulus motion rate.

\section{Secondary endpoints}

(1) Plasma BNP: the first day, the second day, the third day, the fourth day, the fifth day, the sixth day, the seventh day and the sixth month after the operation

(2) Plasma myocardial enzymes: the first day, the second day, the third day, the fourth day, the fifth day, the sixth day, and the seventh day after the operation.

Index includes peripheral plasma myocardial enzyme levels (myoglobin, troponin, creatine kinase, muscle Acid kinase isoenzyme and lactate dehydrogenase).

(3) Heart rate and blood pressure monitoring: the first day, the second day, the third day, the fourth day, the fifth day, the sixth day, the seventh day and the sixth month after the operation.

(4) Canadian angina score: the seventh day, the first month, the second month, the third month, the fourth month, the fifth month and the sixth month after operation.

(5) Clinical adverse events: observed for six months of cardiac adverse events, including death, heart failure and recurrent myocardial infarction.

6) Safety index: the seventh day and the sixth month after operation Index includes liver and kidney function levels.

\section{Safety endpoints}

The safety endpoints mainly include cardiovascular death, nonfatal myocardial infarction, nonfatal stroke and heart failure, liver and kidney function changes (such as serum creatinine, urea nitrogen, and serum uric acid), other laboratory tests (liver function and serum biochemistry) and cardiovascular complications disease.

\section{Statistical methods}


All data will be analyzed with SPSS 22.0 software. If measurement data conforms to a normal distribution, it will be expressed as the mean \pm standard deviation $( \pm S)$. Paired $t$ tests will be used for comparisons of measurements before and after treatment. Multifactor analysis of variance will be used for repeated measurements data. If the measurement data do not conform to a normal distribution, they will be represented by the median (P25, P75). The Wilcoxon rank sum test will be used for comparisons of measurements before and after treatment. The enumeration data will be analyzed by the $\chi^{2}$ test.

\section{Technical Route}

Table 1. 2015 Guidelines for the Diagnosis and Treatment of Acute Myocardial Infarction

\begin{tabular}{|c|c|c|c|c|c|c|c|c|c|}
\hline \multirow[t]{2}{*}{ Test phase } & \multirow[t]{2}{*}{$\begin{array}{l}\text { Screening } \\
\text { period/baseline }\end{array}$} & \multicolumn{2}{|c|}{$\begin{array}{l}\text { Treatment } \\
\text { Period }\end{array}$} & \multicolumn{3}{|c|}{ Follow-up } & \multirow[b]{2}{*}{$\begin{array}{l}\mathrm{D} 90 \\
\pm 7\end{array}$} & \multirow[b]{2}{*}{$\begin{array}{l}\text { D120 } \\
\pm 7\end{array}$} & \multirow[b]{2}{*}{$\begin{array}{l}\text { D180 } \\
\pm 7\end{array}$} \\
\hline & & D1 & $\begin{array}{l}\text { D2- } \\
6\end{array}$ & D7 & $\begin{array}{l}\text { D30 } \\
\pm 7\end{array}$ & $\begin{array}{l}\mathrm{D} 60 \\
\pm 7\end{array}$ & & & \\
\hline Visit & V1 & V2 & V3 & V4 & V5 & V6 & V7 & V8 & V9 \\
\hline $\begin{array}{l}\text { Blood } \\
\text { pressure/heart } \\
\text { rate }\end{array}$ & * & * & * & * & * & * & * & * & * \\
\hline $\begin{array}{l}\text { Physical } \\
\text { examination }\end{array}$ & * & * & * & * & * & * & * & * & * \\
\hline Ultrasonography & & * & & * & & & & & * \\
\hline BNP & & * & * & * & & & & & * \\
\hline $\begin{array}{l}\text { Myocardial } \\
\text { enzyme profile }\end{array}$ & & * & * & * & & & & & \\
\hline $\begin{array}{l}\text { liver function(ALT } \\
\text { AST) }\end{array}$ & & & & * & & & & & * \\
\hline $\begin{array}{l}\text { Renal function(Cr] } \\
\text { BUN口UA) }\end{array}$ & & & & * & & & & & * \\
\hline $\begin{array}{l}\text { Angina pectoris } \\
\text { score }\end{array}$ & & & & * & * & * & * & * & * \\
\hline Adverse events & & * & * & * & * & * & * & * & * \\
\hline
\end{tabular}

\section{Discussion}

The study is designed to observe the effect of ivabradine on acute myocardial infarction patients after $\mathrm{PCl}$ and to explore a new treatment method. We expect that this method will reduce the mortality rate of 
early acute myocardial infarction patients and improve their clinical prognosis and quality of life.

Acute myocardial infarction refers to a sharp decrease or interruption in coronary blood flow and causes severe myocardial ischemia. Thus, it is a serious cardiovascular system disease. Percutaneous coronary intervention is a common treatment method that can restore the blood supply and improve blood flow. However, the incidence of adverse cardiovascular reactions after $\mathrm{PCl}$ is extremely high. One of common factors for adverse events after interventional therapy is increased heart rate.

In the early stage of acute myocardial infarction, the function of the vagus nerve is weakened. The ability to reduce the heart rate and negatively regulate the sinus rhythm are reduced. Consequently, sympathetic nerves are remodeling and lead to an increase in heart rate. The rate of sinus tachycardia is $30 \%-40 \%$ in acute myocardial infarction, especially in anterior myocardial infarction. Increased resting heart rate can aggravate myocardial ischemia and hypoxia and lead to enlargement of the infarct size, moreover, the risk of death increases ${ }^{10}$. Increased heart rate raises myocardial oxygen consumption and decreases coronary perfusion. Consequently, the imbalance between oxygen supply and oxygen demand induces angina symptoms. These symptoms also promote the production of harmful free radicals, which damage the vascular endothelium and lead to the progression of atherosclerosis. Free radicals also increase atherosclerotic plaque rupture and the risk of thrombosis and even cause sudden cardiac death ${ }^{11}$. Researchers have confirmed that the heart rate of coronary heart disease patients, including myocardial infarction patients, must be controlled below 70 beats $/ \mathrm{min}$. The aim of controlling the heart rate is to increase coronary perfusion, reduce myocardial oxygen consumption, prevent angina pectoris and improve clinical outcomes ${ }^{12}$. The results of a clinical study of 1486 people ${ }^{13}$ showed that controlling the ventricular rate could significantly reduce the mortality of patients with acute myocardial infarction during hospitalization and improve the clinical prognosis of patients. Another meta-analysis of 30904 myocardial infarction patients ${ }^{14}$, including 25 randomized controlled clinical trials, showed that with each drop of 10 beats/min in resting heart rate, cardiac death was reduced by $30 \%$. Additionally, the risk of sudden cardiac death was reduced by $39 \%$. In addition, mortality was reduced by $20 \%$. Therefore, actively controlling the ventricular rate has become one of the important methods for the clinical treatment of cardiovascular diseases.

Previous studies have shown that heart failure patients often use ivabradine to improve clinical symptoms. Patients with heart failure are likely to be rehospitalized and die during the first 2 to 3 months after leaving the hospital. Increased heart rate is one of the important factors in adverse cardiovascular events. The CIBIS-II study showed that with each drop of 5 beats/min heart rate, the risk of death was reduced by $8 \%{ }^{15}$. Early use of ivabradine in heart failure patients can reduce ventricular rate and mortality and improve survival. The 2012 European Society of Cardiology (ESC) Heart Failure Treatment Guidelines pointed out that if heart failure patients have a heart rate of $\geq 70$ beats $/ \mathrm{min}$, left ventricular ejection fraction (LVEF) $\leq 35 \%$, and NYHA class IIIIV, it is recommended to administer ivabradine combination therapy to control the ventricular rate ${ }^{16}$. 
In this clinical trial, 500 acute anterior myocardial infarction patients after PCl will be enrolled. Their electrocardiogram must show sinus rhythm and the ventricular rate $\geq 75$ beats $/ \mathrm{min}$. Patients who are intolerant of $\beta$-blockers will be excluded. The patients will be randomly divided into two groups; one group will undergo conventional treatment and the other will undergo conventional treatment plus ivabradine. The patients' systolic blood pressure, heart rate and physical examination will be followed up for 6 months. According to these indexes, investigators will determine the dose of $\beta$-blocker drugs to evaluate whether ivabradine is effective in patients.

Currently, the application of ivabradine in acute myocardial infarction is still being explored. Studies have shown that both ivabradine and $\beta$-blockers can reduce heart rate. However, compared with $\beta$-blockers, ivabradine can cause a prolonged cardiac diastolic period. Furthermore, ivabradine can improve cardiac perfusion and hypoxia without negative effects on cardiac contractility ${ }^{17}$. More importantly, ivabradine is extremely safe. Our research group believes that ivabradine may be a promising treatment method to reduce the mortality of early acute myocardial infarction and improve the clinical prognosis of myocardial infarction patients.

\section{Declarations}

\section{Competing Interests:}

The authors have declared that no competing interests exist.

\section{Funding}

The Shanghai Key Medical Specialties Construction Project (ZK2019A11) ;; Young Elite Scientists Sponsorship Program by CAST(QNRC2-B03) 『Clinical Advantage Discipline of Health System of Putuo District in Shanghai 2019ysxk01) $₫$ Medical innovation research project of Shanghai Science and Technology Commission (20Y11910100)

\section{Consent for publication}

Model consent form and other related documentation that include identifying images or other personal or clinical details of participants are available on reasonable request, only if the participants approve to share their personal or clinical details that compromise anonymity.

\section{Availability of data and materials ;}

After completion of the study, the results will be made public through publication in a scientific journal along with conferences related to pain management, and the clinicaltrials.gov website.

\section{Authors'contributions}


Jun-Qing Gao and Xu Wang contributed equally in drafting and writing of this manuscript. Jun-Qing Gao and Xu Wang should be considered co-first authors.Ling-Yan Li,Hua Zhang,are co-chief investigators who contributed equally in designing and development of this protocol. Hong Zhang and Ying Liu analyzed data ,generated figures. Tian-Jiao Lv,Bing-Yu Han and Ye Jian performed the data interpreatio All authors have read and approved the final manuscript.

\section{References}

1.McAlister, FA; Wiebe, N; Ezekowitz, JA; et al.Meta-analysis: beta-blocker dose, heart rate reduction, and death in patients with heart failure.Ann Intern Med.2009,150(11):784-94

2.Petite SE, Bishop BM, Mauro VF. Role of the Funny Current Inhibitor Ivabradine in Cardiac Pharmacotherapy: A Systematic Review. Am J Ther. 2018 Mar/Apr;25(2):e247-e266

3.Böhm M, Swedberg K, Komajda M, Borer JS, Ford I, Dubost-Brama A, Lerebours G, Tavazzi L, SHIFT Investigators. Heart rate as a risk factor in chronic heart failure (SHIFT): the association between heart rate and outcomes in a randomised placebo-controlled trial. Lancet. 2010 Sep 11;376(9744):886-94

4.Zugck C, Martinka P, Stöckl G. Ivabradine treatment in a chronic heart failure patient cohort: symptom reduction and improvement in quality of life in clinical practice. Adv Ther. 2014 Sep;31(9):961-74.

5.China Heart Failure Diagnosis and Treatment Guidelines 2018(in Chinese).Chinese Journal of Heart Failure and Cardiomyopathy.2018(04):196-225.

6.Barillà F, Pannarale G, Torromeo C, Paravati V, Acconcia MC, Tanzilli G, Mangieri E, Dominici T, Martino F, Pannitteri G, Gaudio C. Ivabradine in Patients with ST-Elevation Myocardial Infarction Complicated by Cardiogenic Shock: A Preliminary Randomized Prospective Study. Clin Drug Investig. 2016 Oct;36(10):849-56

7.Priti K, Ranwa BL, Gokhroo RK, Kishore K, Bisht DS, Gupta S. Ivabradine vs metoprolol in patients with acute inferior wall myocardial infarction-"Expanding arena for ivabradine". Cardiovasc Ther. 2017 Aug;35(4)

8.O'Connor DM, Smith RS, Piras BA, Beyers RJ, Lin D, Hossack JA, French BA. Heart Rate Reduction With Ivabradine Protects Against Left Ventricular Remodeling by Attenuating Infarct Expansion and Preserving Remote-Zone Contractile Function and Synchrony in a Mouse Model of Reperfused Myocardial Infarction. J Am Heart Assoc. 2016 Apr 22;5(4)

9.Cao X, Sun Z, Zhang B, Li X, Xia H. The Effects of Ivabradine on Cardiac Function after Myocardial Infarction are Weaker in Diabetic Rats. Cell Physiol Biochem. 2016;39(5):2055-2064.

10.Kannel WB, Kannel C, Paffenbarger RS Jr, Cupples LA. Heart rate and cardiovascular mortality: the Framingham Study. Am Heart J. 1987 Jun;113(6):1489-94. 
11.Custodis F; Schirmer SH; Baumhäkel M; et al.Vascular pathophysiology in response to increased heart rate.J Am Coll Cardiol.2010,56(24):1973-83

12. Ambrosetti M; Scardina G; Favretto G; et al.Heart rate as a therapeutic target after acute coronary syndrome and in chronic coronary heart disease.G Ital Cardiol (Rome).2017,18(3 Suppl 1):3S-16S

13. Malik, M; Camm, AJ; Janse, MJ; et al.Depressed heart rate variability identifies postinfarction patients who might benefit from prophylactic treatment with amiodarone: a substudy of EMIAT (The European Myocardial Infarct Amiodarone Trial).J Am Coll Cardiol.2000,35(5):1263-75

14. Cucherat, M; Quantitative relationship between resting heart rate reduction and magnitude of clinical benefits in post-myocardial infarction: a meta-regression of randomized clinical trials. Eur Heart J.2007,28(24):3012-9

15. Mulder BA, Damman K, Van Veldhuisen DJ, et al.Heart rate and outcome in heart failure with reduced ejection fraction:differences between atrial fibrillation and sinusrhythm-A CIBIS $\otimes$ analysis.Clin Cardiol.2017;40 (9) :740-5.

16. Borer JS, Böhm M, Ford I, et al.Effect of ivabradine on recurrent hospitalization for worsening heart failure in patients with chronic systolic heart failure:the SHIFT Study.Eur Heart J.2012;33 (11) :2813-20.

17. Seo, Y; Ohte, N; Effects of Heart Rate Reduction by Ivabradine for Heart Failure Beyond $\beta$-Blockers.Circ J.2019,83(10):1991-1993

\section{Figures}




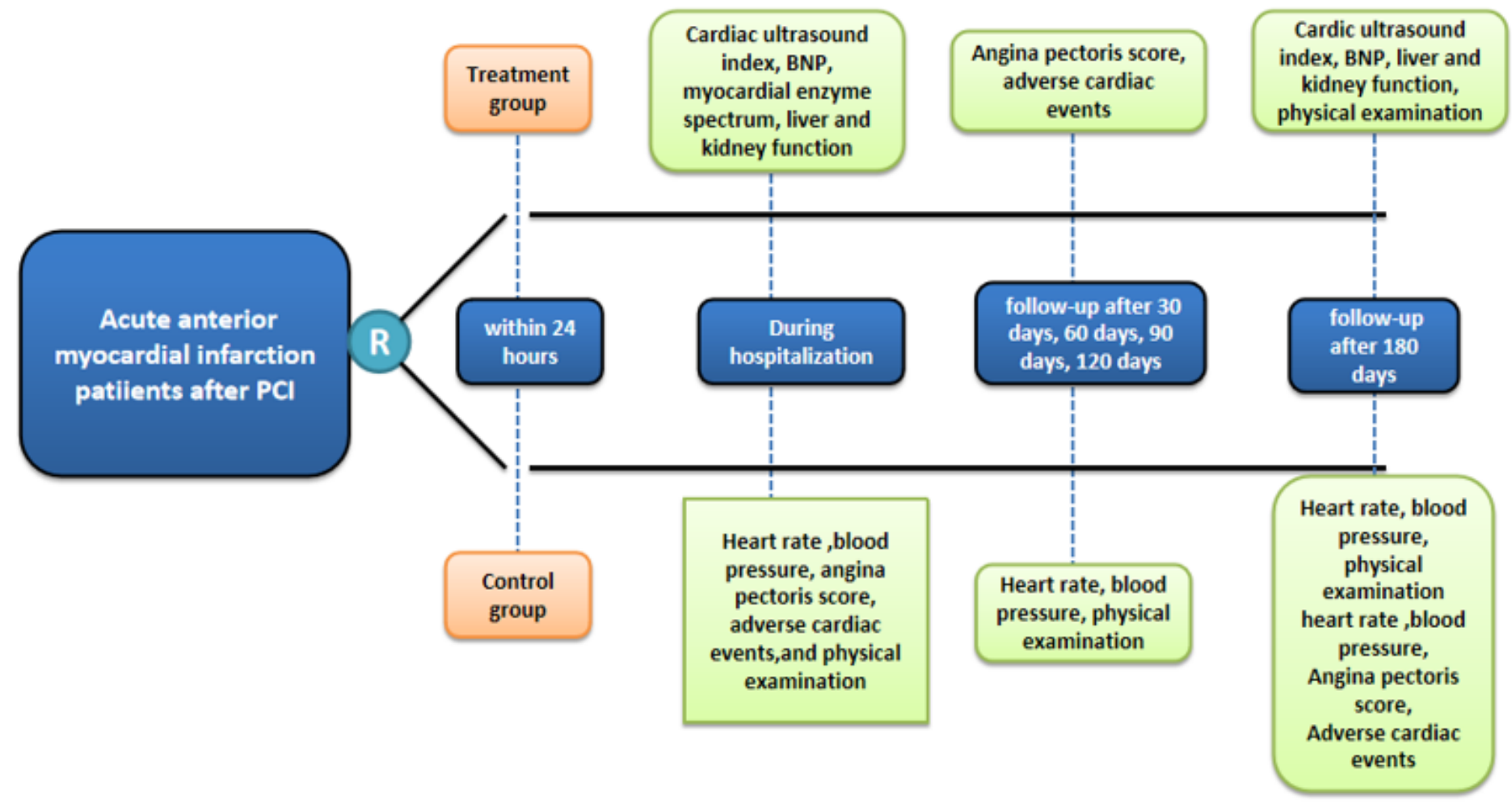

\section{Figure 1}

The Flowdigram.

\section{Supplementary Files}

This is a list of supplementary files associated with this preprint. Click to download.

- spiritfigure.doc 